\title{
Strings and Discrete Fluxes of QCD.
}

\author{
Z. Guralnik \\ University of Pennsylvania \\ Philadelphia PA, 19104 \\ guralnik@ovrut.hep.upenn.edu
}

\begin{abstract}
We study discrete fluxes in four dimensional $S U(N)$ gauge theories with a mass gap by using brane compactifications which give $\mathcal{N}=1$ or $\mathcal{N}=0$ supersymmetry. We show that when such theories are compactified further on a torus, the t'Hooft magnetic flux $m$ is related to the NS two-form modulus $B$ by $B=2 \pi \frac{m}{N}$. These values of $B$ label degenerate brane vacua, giving a simple demonstration of magnetic screening. Furthermore, for these values of $B$ one has a conventional gauge theory on a commutative torus, without having to perform any T-dualities. Because of the mass gap, a generic $B$ does not give a four dimensional gauge theory on a non-commutative torus. The Kaluza-Klein modes which must be integrated out to give a four dimensional theory decouple only when $B=2 \pi \frac{m}{N}$. Finally we show that $2 \pi \frac{m}{N}$ behaves like a two form modulus of the QCD string. This confirms a previous conjecture based on properties of large $N$ QCD suggesting a T-duality invariance.
\end{abstract}




\section{Introduction}

Recently, an improved understanding of the infrared properties of QCD has been gained by studying classical configurations of $M$ theory. In [1] [2] [3], a theory in the same universality class as four dimensional $\mathcal{N}=1$ QCD, known as MQCD, was studied by wrapping an $\mathrm{M}$ theory fivebrane on a holomorphic curve $\Sigma$ embedded in the spacetime $R^{10} \times S^{1} . \mathcal{N}=0$ MQCD was also constructed in [1] by taking $\Sigma$ to be a non-holomorphic two-cycle of minimal area. The results of this paper are applicable to either case. We will further compactify the theory on a torus of finite size, so that the M-fivebrane wraps $\Sigma \times T^{2}$ which is embedded in $R^{8} \times T^{2} \times S^{1}$. In the absence of fundamental matter, $S U(N)$ QCD on a two-torus has a discrete magnetic flux $m$ which is defined modulo $N$ [4]. The supergravity quantities corresponding to discrete Yang-Mills fluxes in the ADS/CFT approach [5] [6] [7] 8] were discussed in [9]. In that context, the discrete fluxes appeared as states in a topological field theory. In the present context, we will see that the 't Hooft flux corresponds to the background M-theory three-form through the relation

$$
\int_{T^{2} \times S^{1}} C=2 \pi \frac{m}{N}
$$

Since this result is independent of the radius of the $S^{1}$, we expect it to persist in the IIA limit, giving

$$
B=\int_{T^{2}} B_{N S}=2 \pi \frac{m}{N}
$$

This relation is peculiar to the case in which there is a mass gap. The background three-form is of course not really discretely quantized. The vacuum energy of the fivebrane has a periodic dependance on this background, with minima at $\int C=2 \pi \frac{m}{N}$. At these minima, there exists a limit in which the low energy theory is QCD, with t' Hooft flux $m$. For other values of $\int C$, one might naively expect to find a gauge theory on a non-commutative torus, [10] but this is not the case. We will show that for $\int C$ away from the minima, it is impossible to decouple the Kaluza Klein modes on $\Sigma$. Interestingly, the absence of a noncommutative deformation is consistent with arguments that the small instanton singularity plays an important role in QCD dynamics [12]. Yang-Mills theories on non-commutative tori do not have a small instanton singularity [13] [14]. For $\Sigma$ corresponding to a theory with a mass gap, the small instanton singularity exists precisely when $\int C=2 \pi \frac{m}{N}$, with $m$ being the 't Hooft flux. If one instead considered a curve $\Sigma$ giving an $\mathcal{N}=2$ Super Yang-Mills theory, a small instanton singularity would only exist 
if $\int C=2 \pi m$, which is gauge equivalent to $\int C=0$. Furthermore, for $\Sigma$ giving $\mathcal{N}=2$ Super Yang-Mills theory, one can decouple the Kaluza-Klein modes for all values of $\int C$. At low energies, we expect a continuous class of four dimensional Yang-Mills theories on a noncommutative torus. The difference between the case with a mass gap and the case without is related to whether or not there is a $U(1)$ gauge field at low energies. Note that the non-commutative star product exists for $U(N)$ theories, but not for $S U(N)$.

The relation (1.1) is a very simple illustration of magnetic screening. 't Hooft has shown that in an electrically confining $S U(N) / Z_{N}$ theory with a mass gap, magnetic fluxes must be light [4]. More precisely, the energy of a magnetic flux must vanish exponentially with the area of the torus. This is simply realized in the M-theory construction. Because of (1.1), the 't Hooft flux is not the central charge associated with a membrane. In fact, the different magnetic fluxes correspond to classically degenerate M-fivebrane configurations. This result differs drastically from what one would obtain by considering a compactification of the M-fivebrane giving $\mathcal{N}=2$ Super Yang-Mills theory. In that case the magnetic flux is related to an additive central charge, rather than an element of $Z_{N}$ labeling degenerate configurations.

We will also argue that $2 \pi \frac{m}{N}$ behaves like a two-form modulus of the QCD string as well as the IIA string. This means that the imaginary part of the action of an MQCD string wrapping the two-torus $n$ times is given by $n \int_{T^{3}} C$. Again, we expect this result to persist in the IIA limit, so that the imaginary part of the action of a wrapped QCD string is given by $2 \pi n \frac{m}{N}$. In the large $N$ limit, this becomes a continuous quantity. Such a relation has been shown explicitly in two dimensions in the large $N$ limit [15] [16] [17]. It was also conjectured to be true in four dimensions on the basis of properties of large $N$ QCD suggesting a T-duality invariance. Such a duality would map,

$$
\tau \rightarrow-\frac{1}{\tau}
$$

where $\tau$ is the Kähler modulus,

$$
\tau=\frac{m}{N}+i \frac{\Lambda^{2} A}{2 \pi}
$$

$A$ is the area of the torus, and $\Lambda^{2}$ is the QCD string tension.

An argument due to 't Hooft states that in an electrically confining $S U(N) / Z_{N}$ theory with a mass gap, magnetic fluxes must be light [4]. More precisely, the energy of a magnetic flux must vanish exponentially with the area of the torus. This is simply realized in the M-theory construction. Because of (1.1), the 't Hooft flux is not the central charge for 
a membrane. In fact, when there is a mass gap, different magnetic fluxes correspond to classically degenerate M-fivebrane configurations. This result differs drastically from what one would obtain by considering a compactification of the M-fivebrane giving $\mathcal{N}=2$ Super Yang-Mills theory. In that case the magnetic flux is related to an additive central charge, rather than an element of $Z_{N}$ labeling degenerate configurations.

In the IIA limit, the M-fivebrane wrapped on $\Sigma$ becomes a configuration of N D4branes suspended between NS fivebranes. For configurations giving $\mathcal{N}=2$ Super YangMills on a commutative torus, the D4-brane worldvolume may also contain integral numbers of fundamental strings, D2 branes and D0 branes [18. However for configurations giving QCD-like theories with a mass gap, the allowed charges are more exotic. There can be no D2 branes or fundamental IIA strings in the low energy theory. This reflects the fact that both the magnetic and electric fluxes in QCD are elements of $Z_{N}$ rather than additive central charges. However there may be Euclidean D0-branes with fractional charge. When the theory is fully compactified on $\Sigma \times T^{4}$, the D0 brane charge has a fractional part of the form $\frac{1}{N} m \wedge m$. This naively appears to violate Dirac quantization in the presence of a single D6 brane. However, due to the nonzero $B=2 \pi \frac{m}{N}$, additional charges are induced on the D6-brane which preserve the Dirac quantization condition.

The organization of this paper is as follows. In section II we briefly review the construction of four dimensional gauge theories by wrapping M-fivebranes on calibrated two-cycles. In section III we perform an additional compactification on a two-torus, allowing a three form modulus in M-theory, and show how this modulus is related to the 't Hooft flux when the theory has a mass gap. In section IV we show how the 't Hooft flux behaves like a two-form modulus for the QCD string on a torus. In section $\mathrm{V}$ we discuss the unusual D-brane charges allowed when there is a mass gap.

\section{M-fivebranes and $\mathrm{QCD}$}

A variety of four dimensional Yang-Mills theories may be obtained by wrapping an M fivebrane on calibrated two cycles embedded in $S^{1} \times R^{10}$ [19]. Here we consider twocycles corresponding to an $\mathrm{SU}(\mathrm{N})$ Yang-Mills theory with a mass gap. For instance a theory in the same universality class as $\mathcal{N}=1 S U(N)$ Yang-Mills is obtained by wrapping the five-brane on a particular holomorphic curve embedded in three complex dimensions. For details of this curve, the reader is referred to [1] [3] [2]. This theory becomes exactly $\mathcal{N}=1, S U(N)$ Yang Mills in the weakly coupled IIA limit in which the radius of the $S^{1}$ 
goes to zero in eleven dimensional Planck units. While taking this limit, the parameters of the curve are adjusted to keep the QCD scale fixed. At the same time one takes the limit $\Lambda_{Q C D} l_{p}^{11} \rightarrow 0$ where $l_{p}^{11}$ is the eleven dimensional Planck length. We will begin by studying the $\mathrm{M}$ theory limit in which the radius of the $S^{1}$ is large. However the quantities which we will compute are discrete and independent of the radius. For our purposes the degree of supersymmetry is unimportant so long as there is a mass gap. Our results are be equally applicable to the theory in the same universality class as pure $\mathcal{N}=0$ QCD [1] which is obtained from a non-holomorphic but minimal area two cycle.

We will make use of two essential properties of two-cycles $\Sigma$ corresponding to $S U(N)$ Yang-Mills theories with a mass gap. The first property is that $H_{1}(\Sigma, Z)$ is generated by a cycle $S^{1 \prime}$ wrapping the $S^{1}$ of space-time N times [1] [3]. The second property is that there are no square integrable harmonic one-forms on $\Sigma$. A compactification of $\Sigma$ obtained by adding points at infinity gives a curve of genus zero [1]. Because of the latter property, there are no light states after dimensional reduction. At low energies, the only solution of the equations of motion for the self dual three-form field strength of the M5-brane is

$$
T^{(3)}=d b^{(2)}-\left.C^{(3)}\right|_{M 5}=0
$$

where $b^{(2)}$ is the two-form gauge potential of the M-fivebrane, and $\left.C^{(3)}\right|_{M 5}$ is the pullback of the bulk three-form potential to the M-fivebrane. Note that if we instead considered a curve $\Sigma$ giving $\mathcal{N}=2$ super Yang-Mills theory, then there would be normalizable harmonic one forms associated with massless $U(1)$ vector multiplets in the low energy theory [19]. If the M-fivebrane worldvolume $X$ has a non-trivial $H_{3}(X, Z)$, there may be several vacua corresponding to solutions of (2.1). We assume that the background $C^{(3)}$ is flat, so $d C^{(3)}=$ 0 . We will see that these vacua are discrete.

\section{Three-form moduli and 't Hooft fluxes}

Let us now compactify $\mathrm{M}$ theory on $S^{1} \times T^{2} \times R^{8}$, and wrap an M-fivebrane on $\Sigma \times T^{2} \times R^{2}$. The low energy theory is an $\mathrm{SU}(\mathrm{N})$ Yang-Mills theory on $T^{2} \times R^{2}$ with a mass gap. The M-fivebrane worldvolume now has non-trivial three cycle $S^{1 \prime} \times T^{2}$. Since the M5 brane theory contains strings coupling to $b^{(2)}$, there is a flux quantization condition

$$
\int_{S^{1 /} \times T^{2}} d b^{(2)}=2 \pi m
$$


where $m$ is an integer. Then because $T^{(3)}=0$,

$$
\left.\int_{S^{11} \times T^{2}} C^{(3)}\right|_{M 5}=2 \pi m
$$

Since $S^{1 \prime}$ wraps $\mathrm{N}$ times around $S^{1}$, the bulk three-form modulus is given by

$$
\int_{S^{1} \times T^{2}} C^{(3)}=2 \pi \frac{m}{N}
$$

In order to get solutions for other values of $\int C^{(3)}$, one must expand $T^{(3)}$ in the Kaluza Klein modes on $\Sigma$. Note that in general $\Sigma$ will change in the presence of a non-zero $T^{3}$, however the property that there are no normalizable harmonic one-forms persists. In this case we do not expect varying $\int C^{(3)}$ to give a continuous class of four dimensional Yang-Mill theories on non-commutative tori.

We will now argue that the the integer $m$ is the 't Hooft flux. To do so we will work in the IIA limit. In this limit the M5-brane becomes a set of $N$ coincident D4-branes stretched between NS5-branes. In the absence of the NS5-branes, the low energy theory would have a $U(N)$ gauge group. However in our case there is a mass gap, and the theory is $S U(N)$. The $U(1)$ degree of freedom is frozen: $\frac{1}{N} \operatorname{tr} F-B_{N S}=0$. This follows from dimensional reduction of $T^{(3)}=0$. If the $\mathrm{D} 4$-brane is compactified on a torus, then fields on the torus are periodic up to gauge transformations $U^{1}$ and $U^{2}$. These gauge transformations are subject to a consistency condition [20],

$$
U^{1}\left(x^{1}, x^{2}\right) U^{2}\left(x^{1}+2 \pi R^{1}, x^{2}\right) U^{1 \dagger}\left(x^{1}+2 \pi R^{1}, x^{2}+2 \pi R^{2}\right) U^{2 \dagger}\left(x^{1}, x^{2}+2 \pi R^{2}\right)=I,
$$

This condition permits the existence of fundamental matter, which appears when strings end on the D4 branes. Note that (3.1) follows from an analogous condition. The U's may be broken up into a $U(1)$ factor and an $S U(N)$ factor, so that the above condition becomes

$$
e^{i \int_{T^{2}} \frac{1}{N} \operatorname{Tr} F} e^{2 \pi i \frac{m}{N}}=I
$$

where $m$ is the $S U(N)$ 't Hooft flux. Since $\frac{1}{N} \operatorname{tr} F=B_{N S}$, we find the 't Hooft flux is precisely the discrete quantum number discovered in the M-theory approach. The fact that the 't Hooft flux is only defined modulo $N$ is reflected in the fact that $2 \pi$ shifts in $\int B_{N S}$ are gauge transformations. Thus the 't Hooft fluxes are associated with degenerate classical vacua of the M5-brane, all of which have $T^{(3)}=0$. This demonstrates magnetic 
screening and is precisely what one expects for for an electrically confining theory with a mass gap [4].

The constraint $F_{U(1)}-B=0$ arising from the existence of a mass gap means that the theory is a standard gauge theory on a commutative torus. In [21][22] [23] this quantity was argued to be a local measure of non-commutativity on the brane worldvolume. A simple way to see that the small instanton singularity is not resolved is to perform a $\mathrm{T}$ duality along one of the cycles of the torus. The $B$ field then becomes the real part of the complex structure of the dual torus. The $N$ D4-branes become a D3-brane at an angle determined by $\frac{1}{N} \operatorname{Tr} F$. Consider wrapping a Euclidean D1-brane on the cycle of the torus on which the T-duality is performed. Because of the relation $B=2 \pi \frac{m}{N}$ relating the real part of the complex structure to the angle of the D3-branes, the D1-brane is at a 90 degree angle to the D3-brane, giving a BPS configuration. There is a Coulomb branch describing motion relative to the D3-branes. The D1 can be placed such that it intersects the D3-branes. Then upon undoing the T-duality, the D1-brane becomes a Euclidean D0brane corresponding to a small instanton. It has been argued [12] that the small instanton singularity plays an important role in QCD dynamics. Our result supports this claim, since a non-commutative deformation does not exist when there is a mass gap. Note that this is related to the freezing of the $U(1)$ degree of freedom. A non-commutative star product does not exist when this degree of freedom is frozen. In $U(N)$ gauge theories on a non-commutative torus, gauge transformations mix the $S U(N)$ and $U(1)$ components.

It is interesting to note that in the $N \rightarrow \infty$ limit of a of the theory with with a mass gap, one is allowed a continuous range of values for $B$, all of which correspond to a gauge theory on a commutative torus.

\section{4. $B$ field for the QCD string}

It has long been suspected that large N QCD is a string theory. We now wish to prove the conjecture [16] [17] that the 't Hooft flux behaves like the real part of a two-form modulus of the QCD-string. To do so we will show that the Euclidean action of a QCD string wrapping $T^{2} n$ times has an imaginary part given by $2 \pi n \frac{m}{N}$ Let us first compute the imaginary part of the action of a wrapped MQCD string. The MQCD string [1] is an open membrane ending on the M5-brane. The worlvolume of the MQCD string is $I \times \mathcal{M}$, where $\mathcal{M}$ is the world sheet of the QCD string and $I$ is an open interval generating the relative homology $H_{1}\left(Y / \Sigma, Z_{N}\right)$. $Y$ is the space in which $\Sigma$ is embedded. For a detailed 
discussion of the MQCD string, the reader is referred to [1] 2]. The action of the open M-twobrane has an imaginary piece:

$$
I m S=\left.\int_{I \times \mathcal{M}} C^{(3)}\right|_{M 2}-\int_{\partial I \times \mathcal{M}} b^{(2)},
$$

where $\left.C^{(3)}\right|_{M 2}$ is the pullback of the bulk three-form to the membrane, and $b^{(2)}$ is the twoform of the M-fivebrane world volume. The second term is necessary for gauge invariance of the open membrane action. Under $C^{(3)} \rightarrow C^{(3)}+d \lambda^{(2)}, b^{(2)}$ transforms to cancel the the non-invariance due to the boundary: $b^{(2)} \rightarrow b^{(2)}+\left.\lambda^{(2)}\right|_{M 2}$. Because $d b^{(2)}-\left.C^{(3)}\right|_{M 5}=0$, we may rewrite (4.1) as

$$
\operatorname{ImS}=\int_{(I-\Omega) \times \mathcal{M}} C^{(3)},
$$

where $\Omega$ is a chain in the M5-brane with boundary $\partial I \times \mathcal{M}$. The MQCD string is homotopic to the IIA string, which is a membrane with the world volume $S^{1} \times \mathcal{M}[1]$. Therefore the above expression becomes

$$
\operatorname{ImS}=\int_{S^{1} \times \mathcal{M}} C^{(3)}
$$

So if $\mathcal{M}$ wraps $T^{2} n$ times,

$$
\operatorname{ImS}=2 \pi n \frac{m}{N}
$$

This result is discrete and independent of the radius of the $S^{1}$, so we expect it to hold in the IIA limit. In the large $N$ limit $\frac{2 \pi m}{N}$ becomes continuous, and behaves as the two-form modulus for the QCD-string, as well as the IIA string.

\section{D-brane charges in theories with a mass gap}

The allowed D-brane charges in theories with a mass gap differ substantially from those without a mass gap. If we had considered a brane construction of an $\mathcal{N}=2$ theory instead, the D2 brane charge and the fundamental string charge would equal the magnetic and electric fluxes respectively. However the fluxes in QCD are very different objects. The term in the M5-brane action of the form $S=\int C^{(3)} \wedge d b^{(2)}$ vanishes in MQCD because $d b^{(2)}-C^{(3)}=0$. Due to the self duality of $d b^{(2)}-C^{(3)}=0$, this term becomes

$$
S=\int C_{R R}^{(3)} \wedge \operatorname{Tr}(F-B)+\int B_{N S} \wedge \operatorname{Tr}^{*}(F-B),
$$

in the IIA limit. This vanishes, so there are no states in the low energy theory carrying either D2 brane or fundamental string charge. 
In brane constructions of QCD, the Euclidean D0-brane charge may be fractional. The importance of such fractional charges for confinement and dynamical generation of superpotentials has been discussed in [12]. We will show that the such fractional charges are consistent with Dirac quantization in the presence of a D6-brane. The Chern-Simons term in the D4 brane action which determines whether there is also D0-brane charge is given by [18

$$
\int C_{R R}^{(1)} \wedge \operatorname{Tr}[(F-B) \wedge(F-B)]
$$

Since $\operatorname{Tr}(F-B)=0$, the D0-brane charge is given by the $S U(N) / Z_{N}$ contribution to the instanton number, which may be fractional [24]. If one compactifies $S U(N) / Z_{N}$ QCD on $T^{4}$, then the instanton number is given by

$$
\frac{1}{16 \pi^{2}} \int \mathcal{F} \wedge \mathcal{F}=\nu+\frac{m \wedge m}{N}
$$

where $\nu$ is an integer and $m$ are the integer 't Hooft fluxes. The fractional piece apparently violates Dirac Quantization in the presence of a single D6-brane, since $Q_{6} Q_{0}^{\prime}$ is not an integer multiple of $2 \pi$. If a D6-brane is present then the theory on the D4 brane contains massive fundamental matter due to string stretched between the D6-brane and the D4brane. Since fundamentals of $S U(N)$ are charged under the $Z_{N}$ center, it would naively seem that 't Hooft flux must vanish modulo $N$ and the instanton number must be integer. However the matter introduced by the D6-brane is in a fundamental representation of $U(N)$, and a nontrivial $S U(N)$ 't Hooft twist may be cancelled by a $U(1)$ twist, as in (3.4). The introduction of fundamental matter by a D6-brane does not prohibit non-trivial 't Hooft fluxes or fractional D0-brane charge.

The Dirac quantization problem is resolved as follows. When the 't Hooft flux is non-zero, there is also a background $\int B$ given by the relation (1.2). For non-zero $\int B$ a D6-brane carries other D-brane charges [18]. The non-integer terms which threaten to violate Dirac quantization turn out to cancel just as they would for a pair of dyons in the presence of a non-zero theta parameter. Suppose that the D4 brane on which the YangMills theory lives is extended in the directions 01234, with the directions 0123 compactified on $T^{4}$. The D4-brane is bounded by NS-5 branes at fixed values of $x^{4}$. Consider the action of a D6-brane with the world-volume $T^{4} \times S^{3}$, where $S^{3}$ is embedded in the directions 56789. Also, suppose that the D6-brane is at a value of $x^{4}$ between the NS5-branes and does not intersect the D4-D0 system. The path integral for the D6 brane contains a term

$$
e^{i\left(\int_{T^{4} \times S^{3}} C_{R R}^{(7)}-\frac{B \wedge B}{4 \pi^{2}} \int_{S^{3}} C_{R R}^{(3)}\right)},
$$


The second term reflects the fact that in the presence of $B$, a D2-brane charge is induced on the D6-brane. A D4-brane charge is induced as well, however the corresponding term in the action does not effect Dirac quantization. This is because the the D4-brane is hodge dual to a D2-brane, and the D2-brane charge vanishes on the D4-brane associated with the Yang-Mills theory. Because of the D4-D0 system, the Ramond-Ramond potentials $C_{R R}^{(7)}$ and $C_{R R}^{(3)}$ are not globally defined. For (5.4) to be well defined, one must have

$$
e^{i\left(\int_{T^{4} \times S^{4}} d C_{R R}^{(7)}-\frac{B \wedge B}{4 \pi^{2}} \int_{S^{4}} d C_{R R}^{(3)}\right)}=1
$$

where $S^{4}$ is embedded in the directions 56789 and surrounds the D4-D0 system, which is a point in 56789. $\int d C^{(7)}$ is simply the D0-brane charge, with fractional piece $\frac{m \wedge m}{N}$. Similarly $\int d C^{(3)}$ is the D4-brane charge, or N. Since $B=2 \pi \frac{m}{N}$, equation (5.5) is satisfied. Roughly speaking, we have found that

$$
Q^{(0)} \wedge Q^{\prime(6)}+Q^{(2)} \wedge Q^{\prime(4)}-Q^{(4)} \wedge Q^{\prime(2)}=2 \pi l,
$$

where the $Q^{\prime}$ charges are associated with the D6-brane, and the $Q$ charges are associated with the D4-branes.

\section{Acknowledgments}

This work was supported in part by DOE under contract No. DE-AC02-76-ER-03071. I am especially grateful to Burt Ovrut and Edward Witten for enlightening conversations. I also profited greatly from discussions with Edna Cheung, Miriam Cvetic, Ori Ganor, Antal Jevicki, Robert de Mello Koch, Morten Krogh, Sanjaye Ramgoolam, and Daniel Waldram 


\section{References}

[1] E. Witten, “Branes and the dynamics of QCD," Nucl. Phys. B507 (1997) 658-690, hep-th/9706109.

[2] A. Hanany, M. Strassler and A. Zaffaroni, "Confinement and strings in MQCD," Nucl. Phys. B513 (1998) 87, hep-th/9707244.

[3] K. Hori and H. Ooguri, "Strong coupling dynamics of four-dimensional N=1 gauge theories from M theory five-brane." Adv. Theor. Math. Phys. 1 (1998) 1, hep-th/9706082.

[4] G. 't Hooft, "A property of electric and magnetic flux in non-abelian gauge theories," Nucl. Phys. B153 (1979) 141.

[5] S. Gubser, I. Klebanov, and A. Polyakov, "Gauge theory correlators from noncritical string theory," Phys. Lett. B428 (1998) 105-114, hep-th/9802109.

[6] J. Maldacena, "The large $N$ limit of superconformal field theories and supergravity," Adv. Theor. Math. Phys. 2 (1998) 231, hep-th/9711200.

[7] E. Witten, "Anti-de-Sitter space and holography," Adv. Theor. Math. Phys. 2 (1998) 253, hep-th/9802150.

[8] E. Witten, "Anti-de-Sitter space, thermal phase transition, and confinement in gauge theories," Adv. Theor. Math. Phys. 2 (1998) 505, hep-th/9803131.

[9] E. Witten, "ADS/CFT correspondence and topological field theory," JHEP 9812 (1998) 012, hep-th/9812012.

[10] A. Connes, M. Douglas, A. Schwarz, "Noncommutative geometry and Matrix theory: compactification on tori," JHEP 9802 (1998) 003, hep-th/9711162.

[11] M. Douglas and C. Hull, "D-branes and the noncommutative torus," JHEP 9802 (1998) 008, hep-th/9711165.

[12] J. Brodie, "Fractional branes, confinement, and dynamically generated superpotentials," Nucl. Phys. B532 (1998) 137, hep-th/9803140.

[13] O. Aharony, M. Berkooz, N. Seiberg, "Light cone description of (2,0) superconformal theories in six-dimensions," Adv. Theor. Math. Phys. 2 (1998) 119, hep-th/9712117.

[14] A. Astashkevich, N. Nekrasov, A. Schwarz, "Instantons on noncommutative $R^{4}$ and (2,0) superconformal six-dimensional theory," Commun. Math. Phys. 198 (1998) 689, hep-th/9810147.

[15] M. Douglas, "Conformal field theory techniques in large N Yang-Mills theory," Cargese Workshop on Strings, Conformal Models and Topological Field Theories, Cargese, France, May 12-26, 1993, hep-th/9311130.

[16] Z. Guralnik, "T-duality of large N QCD," 1998 Paris workshop on Quantum Chromodynamics. hep-th/9903021.

[17] Z. Guralnik, "Duality of large $N$ Yang-Mills theory on $T^{2} \times R^{n}$," hep-th/9804057.

[18] M. Douglas, "Branes within branes," Cargese 1997, Strings, branes and dualities 267, hep-th/9512077. 
[19] E. Witten, "Solutions of four-dimensional field theories via M Theory, Nucl. Phys. B500 (1997) 3, hep-th/9703166.

[20] Z. Guralnik and S. Ramgoolam, "Torons and D-brane bound states," Nucl. Phys. B499 (1997) 241, hep-th/9702099.

[21] F. Ardalan, H. Arfaei and M.M. Sheikh-Jabbari, "Mixed branes and M(atrix) theory on non-commutative torus," hep-th/9803067.

[22] F. Ardalan, H. Arfaei and M.M. Sheikh-Jabbari, "Noncommutative geometry from strings and branes," JHEP 9902 (1999) 016, hep-th/9810072.

[23] C.-S Chu and P.-M Ho, "Noncommutative open string and D-brane," Nucl. Phys. B550 (1999) 151, hep-th/9812219.

[24] G. 'tHooft, "Some twisted self-dual solutions for the Yang-Mills equations on a hypertorus," Comm. Math. Phys 81 (1981) 267. 